\title{
Bridging the Divide: US and Cuban Scientists Speak Up
}

\section{Gail Reed MS}

This roundtable began with world-renowned US and Cuban experts in arboviruses (particularly those transmitted by mosquitoes) but was quickly transformed with the participation of globally recognized scientists in other fields. All have contributed and continue to contribute to the often frustrating efforts to advance bilateral health cooperation, for the benefit of the USA, Cuba and the world. Each from their own vantage point argues why such cooperation can release potential to create scientific synergies capable of addressing some of the most perplexing and urgent global health problems.

MEDICC Review is grateful to them for adding their authoritative and committed voices-as well as their personal stories-to this special issue of the journal. The participants: Peter Agre, Jon Andrus, Juan A. Bisset, María Guadalupe Guzmán, Eva Harris, Eric Martínez and Pedro Valdés. (See brief biographical notes about each at the end of the roundtable.)

MEDICC Review: How have you been involved in US-Cuba joint scientific work? Have you seen concrete results?

Pedro Valdés: In 1969, I met Erwin Roy John, who directed the Brain Research Laboratory at New York University (NYU) Medical Center and was one of the first neuroscientists to use digital computers in animal and human brain studies. That year, he facilitated a donation from a group of New York scientists to what was to become, and is now, the Cuban Neuroscience Center.

This collaboration with NYU was stepped up a year later when Cuba produced its first microcomputer, leading to groundbreaking work on computerized EEG analysis for objective evaluation of brain function and dysfunction. In turn, this resulted in a coauthored article in Science (See John ER, et al. Neurometrics. Science. 1977 Jun 24;196(4297):1393-410).

This kind of collaboration has continued and expanded with colleagues from University of California (San Diego and Los Angeles), as well as Harvard, and has been essential for developing a population-based approach to hierarchical active screening, implemented in Cuba and several other countries. One important result is a Cuban program for early detection of hearing loss through infant screening, a key contribution to pursuing normal development of children with hearing problems.

Peter Agre: I've had the privilege of interacting with Cuban colleagues in multiple areas of research. Each was outstanding in terms of scholarship and superb in terms of collegiality.

It was a great honor to serve as Honorary President of the 2012 Biotechnology Havana with excellent hosting by Professor Manuel Raíces. I also had the wonderful opportunity to serve as plenary lecturer at Quimicuba 2015, organized by Professor Luis Alberto Montero Cabrera, who has subsequently and brilliantly served as Visiting Professor of Chemistry at Johns Hopkins University. Most recently I was part of a Cuba-AAAS Vector-borne Disease Symposium that included members of the Pedro Kourí Tropical
Medicine Institute (IPK) and several US investigators, held in Havana in August 2017.

Jon Andrus: Working under the guidance of Dr Ciro de Quadros, I was a PAHO Regional Advisor for Polio in the Americas. As such, Ciro asked me to coordinate and supervise acute flaccid paralysis (AFP) surveillance in the Americas Region, coordinate the network of polio laboratories, and develop the protocol for certification of polio eradication in the Region.

I had a chance to work with all PAHO member states, including Cuba, on polio eradication issues, especially pertaining to AFP and lab surveillance and the certification committees of each country. I ended up being coauthor of a paper with Cuban scientists, as well as Dr Elizabeth Bell (Mas Lago P, et al. Lessons from Cuba: Mass campaign administration of trivalent oral poliovirus vaccine and seroprevalence of poliovirus neutralizing antibodies. Bull World Health Organ. 1994;72(2):221-5).

During that time, I also worked with Mexican Rotarians from the Yucatán Peninsula to mobilize oral polio vaccine for Cuban children after the disbanding of the former Soviet Union. That way, Cuban children were protected from poliovirus imported from other countries before the Americas' eradication target was achieved.

In 2003, I became Director of PAHO's Immunization team. We conducted reviews of national immunization programs in all countries, including Cuba.

Eva Harris: I've been collaborating with Cuban scientists and participating actively in scientific meetings on the island since 1992. From 1992-2004, I was closely involved with the Genetic Engineering and Biotechnology Center in Havana (CIGB), attending biannual conferences and participating as an instructor in various courses.

While continuing to interact with CIGB, around 2003, I began working more closely with IPK, as my work focused on dengue and other arboviral diseases, aligned with IPK's interests and strengths. In particular, I've collaborated extensively with $\mathrm{Dr}$ María Guadalupe Guzmán, who directs dengue research (and more!) at IPK. From 2005 to now, with PAHO's assistance, I have served as an instructor in the International Dengue Course held every two years in Havana, where I give several presentations on different aspects of my work on the virology, pathogenesis, immunology, epidemiology and control of dengue-and now Zika and chikungunya as well.

To support IPK's excellent scientific work, I've facilitated publications and reagents for Dr Guzmán's group, as well as coauthored papers together, including a major review article on dengue published in The Lancet that Dr Guzmán invited me to coauthor with her. We have also collaborated on international grants/programs sponsored by the WHO-hosted Special Programme for Research and Training in Tropical Diseases (TDR) and the European Union, including revising WHO Guidelines 
for dengue classification and management, as well as various research programs.

María Guadalupe Guzmán: I work in arboviruses, particularly dengue, and now also Zika and chikungunya. Over the years, my research has focused on gaining a deeper understanding of dengue, characterizing the Cuban epidemics, and studying the risk factors associated with the severe form of the disease, as well as diagnosis, vaccine development, pathogenesis and so forth.

At IPK, we've maintained close collaboration over the decades with Professor Scott Halstead, an eminent US scientist in the field, and with other prominent researchers and institutions, resulting in new knowledge and publications. Joint publication, as Eva Harris mentioned, has been particularly important.

We've also developed important collaborative work with the Centers for Disease Control (CDC) of Puerto Rico on aspects of dengue diagnosis and surveillance, more recently applied to Zika and chikungunya, through the good offices of PAHO. Both our institutions are $\mathrm{PAHO} / \mathrm{WHO}$ collaborating centers, and we have worked together to develop these aspects in the region's laboratories through the PAHO-sponsored lab network, RELDA. This includes proficiency tests, reagent donations, exchange of diagnostic protocols, personnel training, and other collaboration.

One of the lines of work with greatest impact has been US scientists' participation in the International Dengue Course in Havana: they have participated in all 15 editions of the course since the first one was organized by IPK in 1987. We have had professors from universities such as Harvard, University of California at Berkeley, Notre Dame and Massachusetts; and from institutions such as CDC and US National Institutes of Health $(\mathrm{NIH})$.

Eric Martínez: IPK has collaborated with various US universities and research institutes from the nineties through 2005, and even before then, related to infectious diseases, mainly HIVIAIDS and other viral diseases. We worked with colleagues at University of North Carolina at Chapel Hill, University of Texas and the University of Buffalo, among others. IPK personnel were also received at CDC, Harvard and Johns Hopkins University.

In April 2003, Harvard's School of Public Health hosted a forum on dengue, where Drs María Guadalupe Guzmán and I attended as guests of the university, while Drs Gustavo Kourí and Juan Bisset were unable to attend since they didn't receive US visas. Over 100 US participants were at the forum, which was followed by a working session with Professor Paul Farmer and Harvard attorneys he had invited, as he said, to find all legal means to collaborate more with Cuba. Dr Farmer and his colleagues have continued to come to Cuba, their last visit in December 2017 for IPK's $80^{\text {th }}$ anniversary.

Juan A. Bisset: As a newly minted IPK researcher, my first bilateral experience was in 1985 when I received a one-year fellowship from WHO to study at the University of California, Riverside, under Dr G.P. Giorghiou. I was trained in detection of vector insecticide resistance and its mechanisms, and the new knowledge acquired contributed to the development of medical entomology labs in Venezuela, Colombia, Perú, Brazil and other countries in the region. It was also the basis for some 100 publications, and had important implications for decision-making on the correct use of insecticides for vector control, mainly for arbovirus vectors in Cuba.

A second experience was the 2006-2013 collaboration with the University of Notre Dame's Professor David W Severson, who sequenced the Aedes aegypti mosquito gene. The first joint WHO-sponsored project with Notre Dame was Molecular Characterization of Insensitive Acetylcholinesterase Mediated Insecticide Resistance in Aedes aegypti from Cuba (2006-2007), which was successfully concluded. It broadened our knowledge base, was published and resulted in important conclusions that informed strategies for insecticide use in Cuba. Professor Severson also participated at least twice in IPK's international dengue course. Another IPK-Notre Dame project was funded by CDRF Global and NIH: Genetic Mapping to Identify the Key Loci Involved in Insecticide Resistance to Aedes aegypti from Cuba (2013-2014). We made some progress but were unable to finish the work due to problems out of our hands related to US-Cuba relations. Yet, researchers persisted with some other joint studies through 2014.

Generally speaking, US-Cuba scientific cooperation in my experience has been quite fruitful, although not exempt from difficulties, primarily associated with the economic, financial, political and social implications of the US government's blockade on Cuba, which at times overrides the will of research centers in both countries to develop collaborative projects.

MEDICC Review: In your field, is bilateral cooperation among researchers, academics and/or clinicians important for population health in the US, Cuba and elsewhere? If so, why?

Peter Agre: The public health of Cubans and citizens of the US and others in the Americas will benefit from close interactions by our scientific leadership. Important trends in infectious disease epidemiology and opportunities in drug development await closer Cuban-US scientific relations.

Cuba is seen as the
bridge for translating
the results of large-
scale global brain
projects into meaning
for the health of people
in resource-scarce
settings

Pedro Valdés: Our current collaborations with colleagues in the US (and internationally) are geared toward developing scalable methods for precision brain health. Cuba is seen as the bridge for translating the results of large-scale global brain projects into meaning for the health of people in resource-scarce settings-as discussed with WHO at the first Global Brain Projects meeting. An example of this approach is the multinational study with Harvard of the long-term effects of malnutrition, using EEG technology. [See article this issueEds.]

Jon Andrus: In communicable disease eradication, infection anywhere is potentially infection everywhere. That's why collaboration is absolutely necessary among all countries of the Americas. This has been important for the Pediatric Dengue Vaccine Initiative (PDVI), but is also critical for measles and rubella. Certainly it also applies to other vaccine-preventable diseases. 
María Guadalupe Guzmán: It's very important. In both countries, we have highly rated scientists and academics who can put themselves at the service of other countries as well. And after all, linking wisdom with experience for the good of humanity is precisely work for wise people!

Eva Harris: In the field of arbovirology, the Cuban experience and expertise is critical, since Dr Guzmán's group at IPK and the late Dr Gustavo Kourí are world renowned for their extensive work in this arena. IPK serves as a PAHO/WHO Collaborating Center for Arboviral Disease, and they play a key role in many international corsortia on arbovirology sponsored by $\mathrm{PAHO}$, the European Union and others. IPK has also led, together with collaborators, pioneering work in community-based participatory approaches to dengue control via control of the mosquito vector, an area in which we have worked extensively as well. All this is very important for research and control of mosquito-borne viral diseases, particularly in Latin America, but also Asia and potentially Africa as well.

More broadly, medical and therapeutic advances led by Cuban researchers and institutions have brought critical new drugs and vaccines to the market, where they have made an important impact.

Eric Martínez: The great importance of this collaboration was demonstrated by bilateral meetings in Havana in late 2016. In particular, I'm referring to the US-Cuba technical consultation on arbovirus research, held October 19 at IPK, aimed at prioritizing dengue vaccine development, gaining broader insight into Zika's natural history and pathogenesis, and preparing a document summarizing the main areas for arbovirus research. Its results were analyzed by the US Secretary of Health and Human Services and Cuba's Minister of Public Health in their meetings October 20-21, in the context of a broader PAHO-sponsored Havana forum involving the entire Americas Region, where immediate collaboration on dengue and Zika were discussed.

A few weeks later (November 28-30), a scientific conference was held in Havana on opportunities for arbovirus research collaboration, specifically to share information on recent findings and priorities for future studies, identifying opportunities for expanded cooperation. [See article this issue-Eds.]

In January 2017, CRDF Global announced its US-Cuba Collaborative Arbovirus Research Initiative, (funded in collaboration with the National Institute of Allergy and Infectious Diseases), calling for joint proposals from US and Cuban researchers. As a result, IPK proposed seven projects with US partners (four of which were approved), covering such important aspects as prenatal Zika infection, early markers for immune activation against Zika, role of prior immunity in dengue infection, and scalability of lessons learned in community participation strategies for prevention of arboviral diseases, among others.

Juan A. Bisset: One of the approved projects Dr Martínez refers to is with the University of Kentucky, preliminary studies of the use of Wolbachia as a strategy to control residual populations of Aedes aegypti and Aedes albopictus in Cuba, coordinated by Dr Stephen Dobson (USA) and Dr María Magdalena Rodríguez (Cuba). The results will be important for control of arbovirus vectors uncontrollable by routine methods and most apt to develop insecticide resistance.
Another project, between Cuba and Johns Hopkins University, with principal investigators Dr Jorge Dimopoulos (USA) and Dr María del Carmen Marquetti (Cuba) concerns Aedes aegypti's competence as a vector for Zika and dengue in Havana, the first research to tackle this issue.

More broadly, Cuba has specialists in medical entomology with vast experience in vector control, and since 1981, has implemented a well-organized national vector control program with trained personnel. The US has experts in this field and advanced technology needed to carry out joint studies. Thus the problem at hand is aligned with the experience, expertise and technology of the two countries to be able to demonstrate the benefits of their joint work for science and for human health.

MEDICC Review: Looking ahead, what projects or areas of bilateral work with colleagues across the Florida Straits do you think are most important for population health in both countries and abroad?

Pedro Valdés: We can combine the cutting-edge brain research of US and Cuban neuroscientists with translational research to provide diagnostics, preventive interventions and treatment options for the huge global burden of brain disorders. To achieve this, in my opinion, the cooperation must go beyond the stage of simple exchange of information or limited joint scientific actions. Funding must be set aside-and barriers to its use eliminated-to seize these unique opportunity for bilateral impact upon global health.

Unfortunately, we often
are forced to wait until
a crisis emerges, and
then we are challenged
to establish basic
scientific collaborations

Peter Agre: Unfortunately, we often are forced to wait until a crisis emerges, and then we are challenged to establish basic scientific collaborations. A case in point is the recent emergence of neural injury alleged to have occurred by US Embassy personnel in Havana, for which alarms have been raised without sharing of information by our health experts. Certainly, more would have been achieved by collaborative efforts by Cuban and US neuroscientists than was accomplished by harsh rhetoric by nonscientific politicians.

Eric Martínez: Vector-borne infections such as dengue and Zika are a priority, to which we must now add the risks of yellow fever, chikungunya, West Nile fever and others. We need to look at clinical management to avoid deaths, viral and immunological pathogenicity, vectors and their control, and also epidemiological surveillance.

Work on vaccines is also an interest in both countries, whether to improve on ones currently available or evaluate efficacy of new vaccines through clinical trials, in which Cuba has recognized experience and success, as well as the infrastructure and trained personnel throughout its national health system.

Other infectious diseases also merit joint research, such as HIVIAIDS, in diagnostics - an area in which Cuba has made contributions-evaluation of new therapies, and social aspects of prevention through health education and changes in risk behaviors, particularly in adolescence. 
Juan A. Bisset: My hope is that the CRDF Global projects approved for joint research can be carried out successfully and their results published internationally to inform national strategies for disease prevention and control of Aedes aegypti; that they generate new opportunities for collaboration and, above all, joint proposals that contribute to the development of science and health in both countries, and produce evidence of value to both science and society throughout the Region.

Jon Andrus: Highly useful areas of collaboration will continue to be the research, including vaccine dose-spacing strategies, and lessons from prevention and control of emerging infections, including arbovirus infections.

\begin{tabular}{|l} 
Every day, we \\
witness emergence \\
or re-emergence of \\
infectious diseases that \\
respect no borders
\end{tabular}

ties, among other factors, are driving this process. Collaboration among countries is an absolute priority in order to diminish the impact and reverse the effects of challenges posed by this global phenomenon.
So the arena of arboviruses becomes a must for cooperation among Cuba, the USA and other countries, centered on research (development of diagnostic tools, vaccines, basic studies to deepen understanding of pathogenicity or protection against disease, epidemiology, etc.), exchange of personnel, bidirectional capacity-building, technological development, and collaboration aimed at improved surveillance and control.

One essential prerequisite is exchange without restrictions-no restrictions on scientific development or access to technology; exchange visits; two-way training programs; organization of courses, symposia, workshops, and joint studies; or access to funding for research.

Eva Harris: There are enormous opportunities to continue and expand collaborations with our Cuban colleagues to enable their excellent and important scientific research in arbovirology and more broadly, human diseases-leading to therapeutics and vaccines with enormous potential for medical application worldwide, including in the USA. I also think it is essential to end the economic embargo, ease travel restrictions, and enable improved internet communication. Cuba has a large cadre of highly trained and extremely capable scientists and clinicians who should play an even greater role on the world stage than they already do. -1 -

\section{ROUNDTABLE PARTICIPANTS}

Peter Agre MD PhD is Bloomberg Distinguished Professor at the Johns Hopkins Bloomberg School of Public Health and Johns Hopkins School of Medicine, and director of the Johns Hopkins Malaria Research Institute. In 2003, Dr Agre, physician and microbiologist, and Roderick MacKinnon were awarded the Nobel Prize in Chemistry for discoveries concerning channels in cell membranes. In 2009, Dr Agre was elected president of the American Association for the Advancement of Science (AAAS) and began actively working for relations with Cuban scientists and their institutions. He led a delegation of US scientists to Havana in December 2009 and has made several trips to the island since then. In 2015, he spoke at the Cuban Academy of Sciences on behalf of the Health Advisory Board of the Bloomberg School of Public Health, as it presented the Academy with a plaque commemorating the scientific collaboration between the Cuban physician Carlos J. Finlay and Jesse W. Lazear, of Johns Hopkins University, in their experiments to prove Finlay's original theories on the agent transmitting yellow fever. Their results opened the way to eliminate this scourge in the tropics.

Jon Kim Andrus MD is professor and director of the Department of Vaccines and Immunization of the University of Colorado's Center for Global Health and professor at George Washington University. Previously, $\mathrm{Dr}$ Andrus served as PAHO deputy director; chief of PAHO's immunization program; and director of polio eradication in WHO's Southeast Asia Region. He began his global health career as a Peace Corps volunteer, serving as a District Medical Officer in
Malawi. Currently, Dr Andrus is co-Chair of the Global Polio Partners Group, and member of the International Monitoring Board for the Polio Transition, PAHO's Technical Advisory Group for Vaccine Preventable Diseases, WHO's South East Asia Regional Verification Commission for Measles and Rubella Elimination, and WHO's Working Group on the Decade of Vaccines. Dr Andrus has published $>120$ scientific peerreviewed papers and has received numerous awards for his leadership in immunization in developing countries. He is Guest Editor of this issue of MEDICC Review.

Juan $\mathbf{A}$. Bisset $\mathrm{PhD}$, is a biologist who has worked since 1981 at IPK, where he heads the Vector Control Department. He is also full professor at the Medical University of Havana. Dr Bisset received postgraduate training at the University of California, Riverside, under $\mathrm{Dr}$ G.P. Giorghiou, in toxicology and the genetics of insecticide resistance in mosquito vectors, a topic he also pursued at the London School of Hygiene and Tropical Medicine in 1990 and 1992. With the 1997 dengue outbreak in Santiago de Cuba, he began research on control of Aedes aegypti. More recently he has become involved in new technologies for arbovirus vector control, including those for dengue, Zika and chikungunya, as well as the use of Wolbachia. Dr Bisset has served as advisor for malaria and dengue control in 7 Latin American countries, received 16 national and international awards, and published 115 scientific articles.

María Guadalupe Guzmán MD PhD DrSc is a virologist at IPK. She has over 30 years' experience working in virology and specifically in dengue and arboviruses. She has directed the PAHO/WHO Collaborating Center for the Study and Control of Dengue since 2005; serves as director of the Center for Research, Diagnostic and Reference Activities at IPK; director of IPK's master's degree program in virology; director of the Cuban project for vaccine development; president of the Cuban Society of Microbiology and Parasitology; and chair of IPK's Scientific Committee. Her work has contributed to knowledge of the pathogenesis, diagnosis, treatment and epidemiology of dengue and dengue hemorrhagic fever, and has developed two new hypotheses concerning dengue. She has been involved in analysis of several dengue epidemics in Cuba and in the Americas region. She is considered an expert by PAHO, WHO, TDR, Dengue Vaccine Initiative, International Council for Science and the European Commision Dr Guzmán has contributed to training of Cuban and Latin American scientists in virology and arboviruses, and has coordinated 15 international dengue courses at IPK. Currently, she coordinates the network of arbovirus laboratories in the Americas region and is a Distinguished Member of the Cuban Academy of Sciences, and a member of the Third World Academy of Science. Dr Guzmán has authored $>300$ papers, short communications and books. She is the author of four patents and is a past member of the Foundation Council of the Global Forum for Health Research and a past PDVI board member.

Eva Harris PhD is a professor at the University of California, Berkeley, in the Division of Infectious Diseases at the School of Public Health, and director of the Center for Global Public Health. 
She has developed a multidisciplinary approach to study the molecular virology, pathogenesis, immunology, epidemiology, clinical presentation and control of dengue, Zika and chikungunya. She has worked in close collaboration with several Latin American countries, particularly Nicaragua, for nearly three decades. In 1997, Dr Harris received a MacArthur Award for work over the previous decade on programs to build scientific capacity in developing countries and address public health and infectious disease issues. Dr Harris was named a Pew Scholar for her work on dengue pathogenesis; received recognition from the Minister of Health of Nicaragua for her contribution to scientific development; and was selected as a Global Leader for Tomorrow by the World Economic Forum. In 2012, she was elected councilor of the American Society of Tropical Medicine and Hygiene and received a Global Citizen Award from the UN Association.

Eric Martínez MD PhD is a pediatrician dedicated to teaching and research on pediatric infectious diseases, particularly dengue, for over three decades. As a PAHO consultant, he has worked in 18 Latin American and Caribbean (LAC) countries during epidemics or as a par- ticipant in courses and seminars on dengue. $\mathrm{He}$ was one of the lead writers of the clinical management in WHO's Dengue: Guidelines for Diagnosis, Treatment, Prevention and Control (2009) and PAHO's Dengue: Guidelines for Patient Care in the Americas (2010). He was the LAC countries' coordinator of the DENCO (dengue control) International Project (clinical diagnosis section) resulting in a proposed new dengue classification, now recommended by WHO. He is also a member of WHO's Data Security Monitoring Board for New Polio Vaccines (Polio Initiative). For many years, he was Deputy Director and Director of two pediatric university hospitals in Havana and headed the Cuban Ministry of Public Health's Department of Medical Research. A decade ago, he joined the IPK teaching staff, focusing mainly on postgraduate education. He also chairs IPK's Research Ethics Committee. Author of $>100$ scientific papers, Dr Martínez has edited 6 books on dengue in Havana, Dominican Republic, Bogotá, Buenos Aires and Rio de Janeiro. He is a member of the Pan American Society of Infectology and the International Society of Infectious Diseases.

Pedro Valdés MD PhD DrSc is the deputy director general for research of the Cuban Neurosci- ences Center, which he cofounded in 1990. His work includes statistical analysis of electrophysiological measurements, neuroimaging (fMRI, EEG and MEG tomography), nonlinear dynamic modeling of brain functions, as well as software and electrophysiological equipment development. $\mathrm{He}$ has been active in promoting brain mapping, setting up international collaborations and societies, and the Cuban and Latin American Brain Mapping projects, participating actively in the Organization for Human Brain Mapping (OHBM) since 1998, having served on its program committee. In 1979 he received postdoctoral training on neurometrics and computational techniques and biophysical modeling of brain electrical activity with Professor E. Roy John at the Brain Research Laboratory of New York University, USA. Dr Valdés is full professor at the Medical University of Havana, member of the Cuban Academy of Sciences and the Latin American Academy of Sciences. He is also Distinguished Professor of Neuroinformatics of the Key Laboratory for Neuroinformation, University of Electronic Science and Technology of China, where he also heads the Joint China-Cuba Laboratory for Frontier Research in Translational Neurotechnology. Dr Valdés serves as OHBM program chair and is a member of MEDICC Review's Editorial Board. 